\title{
The forward kinematics of dual rolling contact of one dual unit sphere on another with dual Darboux frame based equation
}

\author{
Mehmet Aydınalp (D) \\ Department of Mathematics, Manisa Celal Bayar University, Manisa, Turkey
}

\begin{abstract}
In this paper, we investigate the forward kinematics of dual rolling contact motion without sliding of one dual unit sphere $\tilde{S}_{m}^{2}$ on the fixed sphere $\tilde{S}_{f}^{2}$ along their dual spherical curves, which correspond to ruled surfaces generated by the straight lines in the real line space $\mathbb{E}^{3}$. We adopt a dual Darboux frame method to develop instantaneous kinematics of dual rolling motion. We obtain some new kinematic equations of rolling motion of the moving sphere $\tilde{S}_{m}^{2}$ with regards to dual unit vectors, dual rolling velocity, and dual geometric invariants. Namely, the dual translational velocity equation of an arbitrary dual point and the equation of the dual angular velocity on the moving sphere $\tilde{S}_{m}^{2}$ are derived. The equation represented by geometric invariants can be handily generalized to suit arbitrary dual spherical curve on $\widetilde{S}_{m}^{2}$ and can be differentiated to any order.
\end{abstract}

Mathematics Subject Classification (2020). 53A25, 53A17, 14J26

Keywords. dual Darboux frame, dual rolling contact, dual spherical curve, dual unit sphere, ruled surface

\section{Introduction}

The introduction of dual numbers was made by Clifford in 1873 [5]. The set $\mathbb{D}$ containing all dual numbers forms a commutative group under addition. The associative laws hold for multiplication. Dual numbers are distrubutive and form a commutative ring having the numbers $\varepsilon a^{*}$ as divisors of zero over the real number field [16]. P. Kotelnikov [9] and E. Study [14] applied dual numbers to space mechanism by means of the principle of transference. With respect to this principle, there exists a one-to-one correspondence between unit vectors of the unit dual sphere $\tilde{S}^{2}$ and the oriented lines of the line space $\mathbb{E}^{3}$. This means that a spherical curve on the surface of the sphere $\tilde{S}^{2}$ represents a ruled surface in the line space $\mathbb{E}^{3}[16]$. Dual Lorentzian space which is a wider algebraic structure than dual space, the principle of transference between Lorentz spaces and other basic concepts were defined by Uğurlu [15].

Ruled surfaces are defined as a special type of surfaces that can be created by the movement of a straight line following a certain path. For this reason, the concepts such as

Email address: aydinalp64@gmail.com

Received: 19.04.2019; Accepted: 19.06.2021 
dual numbers, dual vectors, dual angle, E. Studys Mapping, etc., are the most fundamental concepts for the use of dual geometry in engineering. Since ruled surfaces are widely used in many fields of science such as moving geometry, mathematical physics, kinematics for modelling the problems and model-based manufacturing of mechanical products, and Computer Aided Geometric Design (CAGD), dual spherical curves on $\tilde{S}^{2}$ become a significant research field of robotics and engineering [1,4,10,13,16-18]. Besides, rolling contact motion is used by many robotic devices such as spherical robots, single wheel robots, and multi-fingered robotic hands to drive from one position and orientation to another [6].

The contact kinematics is given in two classifications in the Euclidean 3 -space $\mathbb{E}^{3}$ : forward kinematics and inverse kinematics. In forward kinematics of rolling contact, the geometry of curves on surfaces is used to observe how the moving surface rolls with time. In inverse kinematics of rolling contact, the desired motion of the moving surface is used to obtain the rolling direction, rolling rate and compensatory spin rate. For the fundamental concepts of kinematics, (see, [3, 6-8,11-13]).

The Darboux frame based kinematics of rolling contact motion was studied by Cui and Dai [6] in Euclidean 3-space. They investigated the forward kinematics of a moving surface making rolling contact motion on a fixed surface. By using the invariants on both surfaces with Darboux frames separately, they obtained new kinematic equations and solved the forward kinematics of rolling contact motion with these equations.

On the other hand, it is well known that thanks to the E. Study mapping or transference principle, the directed lines in line space correspond to dual unit vectors in dual space $[9,14]$. In this context, a connection can be established between a ruled surface in real space and a dual spherical curve in dual space. It is easier to study the theory of curves than the theory of surfaces. This means that we can derive simpler equations. Therefore, it is advantageous to examine the unit spherical curves in dual space representing the ruled surfaces in real space. Besides, we can examine these dual unit spherical curves in two parts as real part and dual part.

In this paper, we apply the theory (in [6]) of the Darboux frame based rolling contact motion to the unit dual spheres $\tilde{S}_{f}^{2}$ and $\tilde{S}_{m}^{2}$ along their dual spherical curves with respect to their dual Darboux frames by determining the relevant ruled surfaces. We extend the rolling contact motion of ruled surfaces in Euclidean 3-space to the rolling contact motion of dual curves on unit dual spheres in dual space. This rolling contact motion of the dual sphere $\tilde{S}_{m}^{2}$ can be called as dual rolling contact motion. Namely, we aim to solve the forward kinematics of dual rolling contact motion of the spheres $\tilde{S}_{m}^{2}$ and $\tilde{S}_{f}^{2}$. Note that at any dual contact point, the sphere $\tilde{S}_{f}^{2}$ is assumed to be fixed and the other $\tilde{S}_{m}^{2}$ is rolling on it and the dual spheres have different dual cartesian coordinate systems. We consider that during the dual rolling contact motion, both of the dual Darboux frames of dual spherical curves are made to coincide at any moment and therefore, the Frenet frames of ruled surfaces are coincident.

\section{Preliminaries}

A dual number is formally expressed as $\bar{a}=\left(a, a^{*}\right)=a+\varepsilon a^{*}$, where $a$ and $a^{*}$ are real part and dual part of the dual number, respectively, and $\varepsilon=(0,1)$ is the dual unit. In this case, the rules are given as follows:

$$
\varepsilon \neq 0, \quad \varepsilon^{2}=0, \quad 0 \varepsilon=\varepsilon 0=0, \quad 1 \varepsilon=\varepsilon 1=\varepsilon .
$$

Let $\mathbb{D}$ denotes the set of all dual numbers. Then the set is given by

$$
\mathbb{D}=\left\{\bar{a}=a+\varepsilon a^{*}: a, a^{*} \in \mathbb{R}, \varepsilon^{2}=0\right\} .
$$


Equality, addition and multiplication operations are defined on $\mathbb{D}$ as

$$
\begin{aligned}
& a+\varepsilon a^{*}=b+\varepsilon b^{*} \Leftrightarrow a=b \text { and } a^{*}=b^{*}, \\
& \left(a+\varepsilon a^{*}\right)+\left(b+\varepsilon b^{*}\right)=(a+b)+\varepsilon\left(a^{*}+b^{*}\right), \\
& \left(a+\varepsilon a^{*}\right)\left(b+\varepsilon b^{*}\right)=a b+\varepsilon\left(a b^{*}+a^{*} b\right),
\end{aligned}
$$

respectively. We should note that $(\mathbb{D},+$, .) is called as a commutative ring with unity. Furthermore, the division of dual numbers $a+\varepsilon a^{*}, b+\varepsilon b^{*} \in \mathbb{D}$ for $b \neq 0$ is defined as follows:

$$
\frac{a+\varepsilon a^{*}}{b+\varepsilon b^{*}}=\frac{a}{b}+\varepsilon\left(\frac{a^{*}}{b}-\frac{a b^{*}}{b^{2}}\right) .
$$

Suppose $f$ denotes a differentiable function with dual variable $\bar{x}=x+\varepsilon x^{*}$. In this case, the expansion of function $f$ in a Maclaurin series can be given as

$$
f(\bar{x})=f\left(x+\varepsilon x^{*}\right)=f(x)+\varepsilon x^{*} f^{\prime}(x),
$$

where $f^{\prime}(x)$ is the derivative of $f(x)$ with respect to $x$. Therefore, we can write

$$
\begin{aligned}
& \cos \bar{x}=\cos \left(x+\varepsilon x^{*}\right)=\cos x-\varepsilon x^{*} \sin x, \\
& \sin \bar{x}=\sin \left(x+\varepsilon x^{*}\right)=\sin x+\varepsilon x^{*} \cos x, \\
& \sqrt{\bar{x}}=\sqrt{x+\varepsilon x^{*}}=\sqrt{x}+\varepsilon \frac{x^{*}}{2 \sqrt{x}}, \quad(x>0) .
\end{aligned}
$$

Let $\mathbb{D}^{3}$ denotes the set of all triples of dual numbers, then

$$
\mathbb{D}^{3}=\left\{\tilde{a}=\left(\bar{a}_{1}, \bar{a}_{2}, \bar{a}_{3}\right) \mid \bar{a}_{i} \in \mathbb{D}, 1 \leq i \leq 3\right\}
$$

and the elements of $\mathbb{D}^{3}$ are called dual vectors. Thus, we may give a dual vector $\tilde{a}$ in the form $\tilde{a}=\vec{a}+\varepsilon \vec{a}^{*}=\left(\vec{a}, \vec{a}^{*}\right)$, where $\vec{a}=\left(a_{1}, a_{2}, a_{3}\right)$ and $\vec{a}^{*}=\left(a_{1}^{*}, a_{2}^{*}, a_{3}^{*}\right)$ are the vectors at $\mathbb{R}^{3}$.

Assume that $\tilde{a}=\vec{a}+\varepsilon \vec{a}^{*}, \tilde{b}=\vec{b}+\varepsilon \vec{b}^{*} \in \mathbb{D}^{3}$ are two dual vectors and $\bar{\lambda}=\lambda+\varepsilon \lambda^{*} \in \mathbb{D}$ is a dual number. In this case, we can write

$$
\begin{aligned}
& \tilde{a}+\tilde{b}=\vec{a}+\vec{b}+\varepsilon\left(\vec{a}^{*}+\vec{b}^{*}\right), \\
& \bar{\lambda} \tilde{a}=\lambda \vec{a}+\varepsilon\left(\lambda \vec{a}^{*}+\lambda^{*} \vec{a}\right) .
\end{aligned}
$$

So, $\mathbb{D}^{3}$ is a unitary $\mathbb{D}$-module with respect to the above operations. It is called $\mathbb{D}$-module or dual space.

Let $\tilde{a}=\vec{a}+\varepsilon \vec{a}^{*}$ and $\tilde{b}=\vec{b}+\varepsilon \vec{b}^{*} \in \mathbb{D}^{3}$ be two dual vectors. Then the inner product and cross product are defined, respectively, as

$$
\langle\tilde{a}, \tilde{b}\rangle=\langle\vec{a}, \vec{b}\rangle+\varepsilon\left(\left\langle\vec{a}, \vec{b}^{*}\right\rangle+\left\langle\vec{a}^{*}, \vec{b}\right\rangle\right)
$$

and

$$
\tilde{a} \times \tilde{b}=\vec{a} \times \vec{b}+\varepsilon\left(\vec{a} \times \vec{b}^{*}+\vec{a}^{*} \times \vec{b}\right),
$$

where $\langle\vec{a}, \vec{b}\rangle$ and $\vec{a} \times \vec{b}$ yield usual scalar and cross products of $\vec{a}$ and $\vec{b}$ in $\mathbb{R}^{3}$, respectively. The norm of a dual vector $\tilde{a}$ is obtained by the formula as follows:

$$
\|\tilde{a}\|=\sqrt{\langle\tilde{a}, \tilde{a}\rangle}=\|\vec{a}\|+\varepsilon \frac{\left\langle\vec{a}, \vec{a}^{*}\right\rangle}{\|\vec{a}\|}, \quad(\vec{a} \neq 0) .
$$

When the norm of $\tilde{a}$ is equal to $1+\varepsilon 0$, it is called a dual unit vector. Besides, a dual unit sphere is expressed as the set that contains all dual unit vectors and given by

$$
\tilde{S}^{2}=\left\{\tilde{a}=\left(\bar{a}_{1}, \bar{a}_{2}, \bar{a}_{3}\right) \in \mathbb{D}^{3} \mid\langle\tilde{a}, \tilde{a}\rangle=1+\varepsilon 0\right\} .
$$

Just as an angle can be assumed as a real number, the dual angle between two dual unit vectors in dual space can also be thought of as a dual number $\bar{a}=a+\varepsilon a^{*}$ in differential 
geometry of spatial mechanisms and in motion analysis. The real part $a$ and the dual part $a^{*}$ of the dual angle denote the projected angle and shortest length between the two oriented lines, respectively.

Dual numbers and dual vectors were used by E. Study in his research on the geometry of lines and kinematics. He deviated great importance to the representation of directed lines with unit dual vectors and presented the mapping which is known by his name as follows:

Theorem 2.1. (E. Studys Mapping): There exists one-to-one correspondence between the dual unit vectors of the dual unit sphere $\tilde{S}^{2}$ and the directed lines of the Euclidean 3-space $\mathbb{E}^{3}$.

(For details of Section 2, see $[2,7,14,16]$ ).

\section{Dual representation of ruled surfaces}

A unit direction vector $\vec{a}$ of the line $L$ and a point $p \in L$ determine an oriented line $L$ in the Euclidean 3-space $\mathbb{E}^{3}$. In this case, if the moment vector $\vec{a}^{*}=\vec{p} \times \vec{a}$ is defined with regards to origin at the Cartesian coordinate system in $\mathbb{E}^{3}$, then the components for the line $L$ can be obtained. The value of $\vec{a}^{*}$ has no dependency on the point $p$. Therefore, one can write as $\vec{q}=\vec{p}+\lambda \vec{a}$ for any other point $q \in L$ and we get

$$
\vec{q} \times \vec{a}=(\vec{p}+\lambda \vec{a}) \times \vec{a}=\vec{p} \times \vec{a}=\vec{a}^{*} .
$$

Thus, an oriented line $L$ is demonstrated by the pair $\left(\vec{a}, \vec{a}^{*}\right)$. On the contrary, any pair $\left(\vec{a}, \vec{a}^{*}\right)$ of vectors satisfying $\langle\vec{a}, \vec{a}\rangle=1,\left\langle\vec{a}, \vec{a}^{*}\right\rangle=0$ describes one oriented line $L$ as

$$
L=\left\{\left(\vec{a} \times \vec{a}^{*}\right)+\lambda \vec{a} \mid \vec{a}, \vec{a}^{*} \in \mathbb{R}^{3}, \lambda \in \mathbb{R}\right\} .
$$

The dual angle $\bar{\theta}=\theta+\varepsilon \theta^{*}$ between two dual unit vectors $\tilde{a}$ and $\tilde{b}$ can be obtained as

$$
\langle\tilde{a}, \tilde{b}\rangle=\cos \bar{\theta}=\cos \theta-\varepsilon \theta^{*} \sin \theta .
$$

If the geometric interpretation of a dual angle is considered, $\theta$ gives the real angle between the lines $L_{1}$ and $L_{2}$ corresponding to the dual unit vectors $\tilde{a}$ and $\tilde{b}$, respectively, and $\theta^{*}$ gives the shortest distance between those lines [2]. The representation and dual geodesic trihedron (dual Darboux frame) of a ruled surface are established by Veldkamp [16]. His procedure can be briefly summarized by as follows:

Suppose $(\tilde{e})$ is a dual curve on the dual unit sphere $\tilde{S}^{2}$ represented by $\tilde{e}(u)=\vec{e}(u)+\varepsilon \vec{e}^{*}(u)$. In this case, the spherical curve determined by the unit vector $\vec{e}$ on (real) unit sphere $S^{2}$ is called the (real) indicatrix of $(\tilde{e})$. Another assumption is that it is not a single point. When the parameter $u$ is given as the arc-length parameter $s$ of the real indicatrix and the differentiation is denoted with respect to $s$ by primes, we get $\left\langle\vec{e}^{\prime}, \vec{e}^{\prime}\right\rangle=1$, where $\vec{e}^{\prime}=\vec{t}$ is the unit tangent vector of the indicatrix. It can be considered that there exists an equation $\vec{e}^{*}(s)=\vec{p}(s) \times \vec{e}(s)$ with infinite solution for the function $\vec{p}(s)$. When we take a solution of the equation as $\vec{p}_{o}(s)$, the set of all solutions of the equation has the form $\vec{p}(s)=\vec{p}_{o}(s)+\lambda(s) \vec{e}(s)$, where $\lambda$ is a real scalar function of $s$. In this case, $\left\langle\vec{p}^{\prime}, \vec{e}^{\prime}\right\rangle=\left\langle\vec{p}_{o}^{\prime}, \vec{e}^{\prime}\right\rangle+\lambda$ is obtained. If $\lambda=\lambda_{o}=-\left\langle\vec{p}_{o}^{\prime}, \vec{e}^{\prime}\right\rangle$ is taken, then one can see that $\vec{p}_{o}(s)+\lambda_{o}(s) \vec{e}(s)=\vec{c}(s)$ is the unique solution for $\vec{p}(s)$ with $\left\langle\vec{c}^{\prime}, \vec{e}^{\prime}\right\rangle=0$. We should note that $\vec{c}(s)$ is also the position vector of the striction curve. The dual curve ( $\tilde{e})$ corresponding to the ruled surface $X_{e}(s, v)=\vec{c}(s)+v \vec{e}(s)$, can be written by $\tilde{e}(s)=\vec{e}+\varepsilon \vec{c} \times \vec{e}$, where

$$
\langle\vec{e}, \vec{e}\rangle=1, \quad\left\langle\vec{e}^{\prime}, \vec{e}^{\prime}\right\rangle=1,\left\langle\vec{c}^{\prime}, \vec{e}^{\prime}\right\rangle=0 .
$$

Then we have

$$
\left\|\tilde{e}^{\prime}\right\|=1+\varepsilon \operatorname{det}\left(\vec{c}^{\prime}, \vec{e}, \vec{t}\right)=1+\varepsilon \Delta,
$$


where $\Delta=\operatorname{det}\left(\vec{c}^{\prime}, \vec{e}, \vec{t}\right)$. Likewise, the dual curve $(\tilde{e})$ has the dual arc-length $\bar{s}$ given as follows:

$$
\bar{s}=\int_{0}^{s}\left\|\tilde{e}^{\prime}(u)\right\| d u=\int_{0}^{s}(1+\varepsilon \Delta) d u=s+\varepsilon \int_{0}^{s} \Delta d u .
$$

Consequently, $\bar{s}^{\prime}=1+\varepsilon \Delta$ is obtained. Thus, the dual unit tangent to the dual curve $(\tilde{e})$ is

$$
\frac{d \tilde{e}}{d \bar{s}}=\frac{\tilde{e}^{\prime}}{\bar{s}^{\prime}}=\frac{\tilde{e}^{\prime}}{1+\varepsilon \Delta}=\tilde{t}=\vec{t}+\varepsilon(\vec{c} \times \vec{t}) .
$$

Lastly, from the definition of dual unit vector $\tilde{g}=\tilde{e} \times \tilde{t}=\vec{g}+\varepsilon(\vec{c} \times \vec{g})$, we can construct the dual frame $\{\tilde{e}, \tilde{t}, \tilde{g}\}$ which is called dual Darboux frame (dual geodesic trihedron) of $X_{e}$ (or $(\tilde{e})$ ). Furthermore, the real orthonormal frame $\{\vec{e}, \vec{t}, \vec{g}\}$ along the striction curve (c) of the ruled surface is called the Frenet frame of ruled surface $X_{e}$. The derivatives of the vectors forming the frame $\{\vec{e}, \vec{t}, \vec{g}\}$ can be presented as follows:

$$
\vec{e}^{\prime}=\vec{t}, \quad \vec{t}^{\prime}=\gamma \vec{g}-\vec{e}, \quad \vec{g}^{\prime}=-\gamma \vec{t}
$$

where $\gamma$ is called the conical curvature [8]. The derivatives of the dual vectors forming the the dual Darboux frame $\{\tilde{e}, \tilde{t}, \tilde{g}\}$ can be presented as follows:

$$
\frac{d \tilde{e}}{d \bar{s}}=\tilde{t}, \frac{d \tilde{t}}{d \bar{s}}=\bar{\gamma} \tilde{g}-\tilde{e}, \frac{d \tilde{g}}{d \bar{s}}=-\bar{\gamma} \tilde{t}
$$

where

$$
\bar{\gamma}=\gamma+\varepsilon(\delta-\gamma \Delta), \delta=\left\langle\vec{c}^{\prime}, \vec{e}\right\rangle .
$$

In this case, the dual Darboux vector of $\{\tilde{e}, \tilde{t}, \tilde{g}\}$ is $\tilde{d}=\bar{\gamma} \tilde{e}+\tilde{g}$. By using the equality of $\Delta$ and the equations in (3.3), we get

$$
\vec{c}^{\prime}=\delta \vec{e}+\Delta \vec{g} .
$$

The dual curve (ruled surface) $\tilde{e}(s)$ has dual curvature given by

$$
\bar{R}=\frac{1}{\sqrt{\left(1+\bar{\gamma}^{2}\right)}} .
$$

When we transform the dual Darboux vector $\tilde{d}=\bar{\gamma} \tilde{e}+\tilde{g}$ into a unit vector $\tilde{d}_{o}$, we get

$$
\tilde{d}_{o}=\frac{\bar{\gamma}}{\sqrt{\left(1+\bar{\gamma}^{2}\right)}} \tilde{e}+\frac{1}{\sqrt{\left(1+\bar{\gamma}^{2}\right)}} \tilde{g} .
$$

If $\bar{\rho}$ denotes the dual spherical radius of curvature, then the dual angle between the dual unit vectors $\tilde{d}_{o}$ and $\tilde{e}$ yield

$$
\cos \bar{\rho}=\frac{\bar{\gamma}}{\sqrt{\left(1+\bar{\gamma}^{2}\right)}}, \quad \sin \bar{\rho}=\frac{1}{\sqrt{\left(1+\bar{\gamma}^{2}\right)}} .
$$

Thus, $\bar{R}=\sin \bar{\rho}$ and $\bar{\gamma}=\cot \bar{\rho}$.

\section{The forward kinematics of rolling contact of dual unit spheres}

In this section, we study the forward kinematics of dual rolling contact motion of dual unit spheres $\tilde{S}_{f}^{2}$ and $\tilde{S}_{m}^{2}$ by applying the dual Darboux frame method in dual space. The main contribution of this section is that a new equation of the dual angular velocity of the rolling motion of a moving sphere $\tilde{S}_{m}^{2}$ on a fixed sphere $\tilde{S}_{f}^{2}$ is formed. The new formulation is specified with respect to three dual unit vectors and dual geometric invariants, which are dual arc lengths of the dual contact curves and the dual induced curvature of these two spheres. 


\subsection{The kinematics of dual rolling contact motion}

In this subsection, we give the dual geometric kinematics of rolling motion of two contact dual unit spheres. We note that during the dual rolling contact motion, both of the spheres have the same dual unit normal vectors at the dual contact point. Let denote the dual contact point by $\tilde{P}$ and denote the fixed sphere and moving sphere at $\tilde{P}$ by $\tilde{S}_{f}^{2}$ and $\tilde{S}_{m}^{2}$, respectively. We should define two different dual cartesian coordinate systems for each sphere, when they undergo dual rolling contact motion. We assume that $\tilde{S}_{f}^{2}$ and $\tilde{S}_{m}^{2}$ are established in the coordinate systems $(\tilde{x}, \tilde{y}, \tilde{z})$ with origin $\tilde{O}$ and $\left(\tilde{x}_{m}, \tilde{y}_{m}, \tilde{z}_{m}\right)$ with origin $\tilde{O}_{m}$, respectively.

Now, suppose $(\tilde{e})$ and $\left(\tilde{e}_{m}\right)$ are two dual contact curves on $\tilde{S}_{f}^{2}$ and $\tilde{S}_{m}^{2}$, respectively. Let us denote the dual Darboux frames attached to the dual contact point $\tilde{P}$ of the dual curves $(\tilde{e})$ and $\left(\tilde{e}_{m}\right)$ as $\{\tilde{e}, \tilde{t}, \tilde{g}\}$ and $\left\{\tilde{e}_{m}, \tilde{t}_{m}, \tilde{g}_{m}\right\}$, respectively. By rolling constraints, the dual vectors $\tilde{t}$ and $\tilde{t}_{m}$ are always collinear and, consequently, are $\tilde{e}$ and $\tilde{e}_{m}$. Therefore, the two dual frames can always be made to coincide at the dual contact point $\tilde{P}$ where $\tilde{e}$ points outward of the $\tilde{S}_{f}^{2}$ and $\tilde{e}_{m}$ points inward of the $\tilde{S}_{m}^{2}$.

Let $\bar{s}$ and $\bar{s}_{m}$ be the dual arc lengths of the dual curves $(\tilde{e})$ and $\left(\tilde{e}_{m}\right)$, respectively. Then the derivative formulas of the dual Darboux frames $\{\tilde{e}, \tilde{t}, \tilde{g}\}$ and $\left\{\tilde{e}_{m}, \tilde{t}_{m}, \tilde{g}_{m}\right\}$ are

$$
\frac{d \tilde{p}}{d \bar{s}}=\tilde{t}, \quad \frac{d}{d \bar{s}}\left[\begin{array}{c}
\tilde{e} \\
\tilde{t} \\
\tilde{g}
\end{array}\right]=\left[\begin{array}{ccc}
0 & 1 & 0 \\
-1 & 0 & \bar{\gamma} \\
0 & -\bar{\gamma} & 0
\end{array}\right]\left[\begin{array}{c}
\tilde{e} \\
\tilde{t} \\
\tilde{g}
\end{array}\right]
$$

and

$$
\frac{d \tilde{p}_{m}}{d \bar{s}_{m}}=\tilde{t}_{m}, \quad \frac{d}{d \bar{s}_{m}}\left[\begin{array}{l}
\tilde{e}_{m} \\
\tilde{t}_{m} \\
\tilde{g}_{m}
\end{array}\right]=\left[\begin{array}{ccc}
0 & 1 & 0 \\
-1 & 0 & \bar{\gamma}_{m} \\
0 & -\bar{\gamma}_{m} & 0
\end{array}\right]\left[\begin{array}{c}
\tilde{e}_{m} \\
\tilde{t}_{m} \\
\tilde{g}_{m}
\end{array}\right]
$$

where $\tilde{p}$ and $\tilde{p}_{m}$ are the dual position vectors of $\tilde{P}$ with respect to the dual Darboux frames $\{\tilde{e}, \tilde{t}, \tilde{g}\}$ and $\left\{\tilde{e}_{m}, \tilde{t}_{m}, \tilde{g}_{m}\right\}$, respectively.

Now, let $\tilde{Q}$ denote an arbitrary dual point on $\tilde{S}_{m}^{2}$ and $\tilde{q}_{m}$ denote the dual position vector of $\tilde{Q}$ in the dual frame $\left\{\tilde{e}_{m}, \tilde{t}_{m}, \tilde{g}_{m}\right\}$ given by

$$
\tilde{q}_{m}=\tilde{p}_{m}+\bar{\lambda}_{1} \tilde{e}_{m}+\bar{\lambda}_{2} \tilde{t}_{m}+\bar{\lambda}_{3} \tilde{g}_{m}
$$

where $\bar{\lambda}_{1}, \bar{\lambda}_{2}$ and $\bar{\lambda}_{3}$ are dual functions. Differentiating $\tilde{q}_{m}$ with respect to $\bar{s}_{m}$ gives

$$
\frac{d \vec{q}_{m}}{d \bar{s}_{m}}=\left(\frac{d \bar{\lambda}_{1}}{d \bar{s}_{m}}-\bar{\lambda}_{2}\right) \tilde{e}_{m}+\left(1+\bar{\lambda}_{1}+\frac{d \bar{\lambda}_{2}}{d \bar{s}_{m}}-\bar{\lambda}_{3} \bar{\gamma}_{m}\right) \tilde{t}_{m}+\left(\bar{\lambda}_{2} \bar{\gamma}_{m}+\frac{d \bar{\lambda}_{3}}{d \bar{s}_{m}}\right) \tilde{g}_{m}
$$

where $\bar{\gamma}_{m}$ is dual spherical curvature function at the dual point $\tilde{Q}$ of $\tilde{S}_{m}^{2}$. Since $\tilde{Q}$ is a fixed dual point of $\tilde{S}_{m}^{2}$, then $\frac{d \tilde{q}_{m}}{d \bar{s}_{m}}=0$. Substituting this into (4.3) gives

$$
\frac{d \bar{\lambda}_{1}}{d \bar{s}_{m}}=\bar{\lambda}_{2}, \quad \frac{d \bar{\lambda}_{2}}{d \bar{s}_{m}}=-1-\bar{\lambda}_{1}+\bar{\lambda}_{3} \bar{\gamma}_{m}, \quad \frac{d \bar{\lambda}_{3}}{d \bar{s}_{m}}=-\bar{\lambda}_{2} \bar{\gamma}_{m} .
$$

Now, let $\tilde{q}$ denote the dual position vector of $\tilde{Q}$ in the dual frame $\{\tilde{e}, \tilde{t}, \tilde{g}\}$ given by

$$
\tilde{q}=\tilde{p}+\bar{\mu}_{1} \tilde{e}+\bar{\mu}_{2} \tilde{t}+\bar{\mu}_{3} \tilde{g}
$$

where $\bar{\mu}_{1}, \bar{\mu}_{2}$ and $\bar{\mu}_{3}$ are dual functions. Differentiating $\tilde{q}$ with respect to $\bar{s}$ gives

$$
\frac{d \tilde{q}}{d \bar{s}}=\left(\frac{d \bar{\mu}_{1}}{d \bar{s}}-\bar{\mu}_{2}\right) \tilde{e}+\left(1+\bar{\mu}_{1}+\frac{d \bar{\mu}_{2}}{d \bar{s}}-\bar{\mu}_{3} \bar{\gamma}\right) \tilde{t}+\left(\bar{\mu}_{2} \bar{\gamma}+\frac{d \bar{\mu}_{3}}{d \bar{s}}\right) \tilde{g}
$$

where $\bar{\gamma}$ is dual spherical curvature function at the dual point $\tilde{Q}$ of $\tilde{S}_{f}^{2}$. By the constraints for dual rolling contact, two dual contact curves have the same dual arc lengths at the 
dual contact point. Since the dual Darboux frames $\{\tilde{e}, \tilde{t}, \tilde{g}\}$ and $\left\{\tilde{e}_{m}, \tilde{t}_{m}, \tilde{g}_{m}\right\}$ are made to coincide at any moment, it follows that

$$
\bar{\lambda}_{1}=\bar{\mu}_{1}, \quad \bar{\lambda}_{2}=\bar{\mu}_{2}, \bar{\lambda}_{3}=\bar{\mu}_{3} .
$$

Then,

$$
\frac{d \bar{\lambda}_{1}}{d \bar{s}_{m}}=\frac{d \bar{\mu}_{1}}{d \bar{s}}, \quad \frac{d \bar{\lambda}_{2}}{d \bar{s}_{m}}=\frac{d \bar{\mu}_{2}}{d \bar{s}}, \quad \frac{d \bar{\lambda}_{3}}{d \bar{s}_{m}}=\frac{d \bar{\mu}_{3}}{d \bar{s}} .
$$

Substituting (4.4) and (4.6) into (4.5) gives

$$
\frac{d \tilde{q}}{d \bar{s}}=\left(\bar{\mu}_{3} \bar{\gamma}_{i}\right) \tilde{t}-\left(\bar{\mu}_{2} \bar{\gamma}_{i}\right) \tilde{g}
$$

where $\bar{\gamma}_{i}=\bar{\gamma}_{m}-\bar{\gamma}$ is induced dual spherical curvature function.

\subsection{Dual velocity formulation of dual rolling contact motion}

The dual velocity of an arbitrary dual point $\tilde{Q}$ on $\tilde{S}_{m}^{2}$ in terms of dual parameter $\bar{t}$ can be obtained from (4.7) as follows:

$$
\tilde{v}_{\tilde{Q}}=\frac{d \tilde{q}}{d \bar{s}} \frac{d \bar{s}}{d \bar{t}}=\bar{\sigma}\left(\bar{\mu}_{3} \bar{\gamma}_{i}\right) \tilde{t}-\bar{\sigma}\left(\bar{\mu}_{2} \bar{\gamma}_{i}\right) \tilde{g}
$$

where $\bar{\sigma}=\frac{d \bar{s}}{d \bar{t}}$ is the magnitude of dual rolling velocity. This equation gives the dual Darboux-frame-based translational velocity formulation of an arbitrary dual point. Let the dual angular velocity of $\tilde{S}_{m}^{2}$ relative to $\tilde{S}_{f}^{2}$ be

$$
\tilde{d}=\bar{d}_{1} \tilde{e}+\bar{d}_{2} \tilde{t}+\bar{d}_{3} \tilde{g} .
$$

When the dual vector $\tilde{r}_{\tilde{P} \tilde{Q}}=\bar{\mu}_{1} \tilde{e}+\bar{\mu}_{2} \tilde{t}+\bar{\mu}_{3} \tilde{g}$ is given, the dual velocity of the dual point $\tilde{Q}$ can be obtained as

$$
\tilde{v}_{\tilde{Q}}=\tilde{d} \times \tilde{r}_{\tilde{P} \tilde{Q}}=\left(\bar{d}_{2} \bar{\mu}_{3}-\bar{d}_{3} \bar{\mu}_{2}\right) \tilde{e}+\left(-\bar{d}_{1} \bar{\mu}_{3}+\bar{d}_{3} \bar{\mu}_{1}\right) \tilde{t}+\left(\bar{d}_{1} \bar{\mu}_{2}-\bar{d}_{2} \bar{\mu}_{1}\right) \tilde{g} .
$$

If we take $\bar{d}_{2}=0$ into consideration from the definition of dual Darboux vector and compare (4.8) with (4.10), the solution of the equations system yields

$$
\bar{d}_{1}=-\bar{\sigma} \bar{\gamma}_{i}, \quad \bar{d}_{2}=0, \quad \bar{d}_{3}=0 .
$$

The dual angular velocity of $\tilde{S}_{m}^{2}$ rolling on $\tilde{S}_{f}^{2}$ is obtained from (4.9) and (4.11) as

$$
\tilde{d}=-\bar{\sigma} \bar{\gamma}_{i} \tilde{e} .
$$

Consequently, the forward kinematics of dual rolling contact motion of $\tilde{S}_{m}^{2}$ relative to $\tilde{S}_{f}^{2}$ with the dual Darboux frame method is completed.

Now, let give an example to understand this method.

\subsection{Example}

In this subsection, we give an example of dual Darboux frame based forward kinematics of one dual unit sphere rolling on another along their dual spherical curves by determining the relevant ruled surfaces. In this example, we consider that dual curve denoted by $(\tilde{e})$ on the fixed dual unit sphere denoted by $\tilde{S}_{f}^{2}$ corresponds to one-sheeted hyperboloid and dual curve denoted by $\left(\tilde{e}_{m}\right)$ on the moving dual unit sphere denoted by $\tilde{S}_{m}^{2}$ (rolling on $\tilde{S}_{f}^{2}$ ) corresponds to cone. To understand the example better, we present some pictures drawn with Mathematica computer program.

Let take the parametrization of one-sheeted hyperboloid as

$$
X(t, v)=(\cos t, \sin t, 0)+v(-\sin t, \cos t, 1) .
$$


where the striction curve is $c(t)=(\cos t, \sin t, 0)$. Also, let denote the Frenet frame of $X(t, v)$ as $\{\vec{e}, \vec{t}, \vec{g}\}$ along the striction curve $(c)$. Then we have

$$
\begin{aligned}
& \vec{e}(t)=\frac{1}{\sqrt{2}}(-\sin t, \cos t, 1), \\
& \vec{t}(t)=(-\cos t,-\sin t, 0), \\
& \vec{g}(t)=\frac{1}{\sqrt{2}}(\sin t,-\cos t, 1),
\end{aligned}
$$

where $\left\|\vec{e}^{\prime}(t)\right\|=\frac{1}{\sqrt{2}}$. When the arc-length of $(c)$ is given as $s$, we have $s=\int_{0}^{t}\left\|\vec{e}^{\prime}(u)\right\| d u=\frac{t}{\sqrt{2}}$. Now, we can write the striction curve $(c)$ and $\{\vec{e}, \vec{t}, \vec{g}\}$ elements with respect to the arclength $s$ as

$$
c(s)=(\cos \sqrt{2} s, \sin \sqrt{2} s, 0)
$$

and

$$
\begin{aligned}
& \vec{e}(s)=\frac{1}{\sqrt{2}}(-\sin \sqrt{2} s, \cos \sqrt{2} s, 1), \\
& \vec{t}(s)=(-\cos \sqrt{2} s,-\sin \sqrt{2} s, 0), \\
& \vec{g}(s)=\frac{1}{\sqrt{2}}(\sin \sqrt{2} s,-\cos \sqrt{2} s, 1),
\end{aligned}
$$

respectively. Furthermore, It can be easly seen that the position vector of the striction curve is $\vec{c}(s)=(\cos \sqrt{2} s, \sin \sqrt{2} s, 0)$.

Now, let denote the invariants of one-sheeted hyperboloid as $\gamma, \delta$ and $\Delta$. We obtain these invariants as follows, respectively:

Via the derivative formulas of the Darboux frame $\{\vec{e}, \vec{t}, \vec{g}\}$, the conical curvature is obtained as $\gamma(s)=\left\langle\vec{t}^{\prime}(s), \vec{g}(s)\right\rangle=1$. Via the derivative of $\vec{c}(s)$, the other two functions are obtained as $\delta(s)=\left\langle\vec{c}^{\prime}(s), \vec{e}(s)\right\rangle=1$ and $\Delta(s)=\operatorname{det}\left(\vec{c}^{\prime}(s), \vec{e}(s), \vec{t}(s)\right)=-1$. By using Eqs. (4.13), the moment vectors are obtained as follows:

$$
\begin{aligned}
& \vec{e}^{*}(s)=\vec{c}(s) \times \vec{e}(s)=\frac{1}{\sqrt{2}}(\sin \sqrt{2} s,-\cos \sqrt{2} s, 1), \\
& \vec{t}^{*}(s)=\vec{c}(s) \times \vec{t}(s)=(0,0,0), \\
& \vec{g}^{*}(s)=\vec{c}(s) \times \vec{g}(s)=\frac{1}{\sqrt{2}}(\sin \sqrt{2} s,-\cos \sqrt{2} s,-1) .
\end{aligned}
$$

From the Eqs. (4.13) and (4.14) the dual unit vectors correspond to one-sheeted hyperboloid are obtained as

$$
\begin{aligned}
& \tilde{e}(s)=\frac{1}{\sqrt{2}}(-\sin \sqrt{2} s, \cos \sqrt{2} s, 1)+\varepsilon \frac{1}{\sqrt{2}}(\sin \sqrt{2} s,-\cos \sqrt{2} s, 1), \\
& \tilde{t}(s)=(-\cos \sqrt{2} s,-\sin \sqrt{2} s, 0), \\
& \vec{g}(s)=\frac{1}{\sqrt{2}}(\sin \sqrt{2} s,-\cos \sqrt{2} s, 1)+\varepsilon \frac{1}{\sqrt{2}}(\sin \sqrt{2} s,-\cos \sqrt{2} s,-1),
\end{aligned}
$$

where $(\tilde{e})$ is the dual curve on $\tilde{S}_{f}^{2}$. From (3.3), the dual curvature of one-sheeted hyperboloid is obtained as

$$
\bar{\gamma}=\gamma+\varepsilon(\delta-\gamma \Delta)=1+2 \varepsilon .
$$

We assume that $\tilde{S}_{f}^{2}$ is established in the fixed coordinate system $(\tilde{x}, \tilde{y}, \tilde{z})$ with origin $\tilde{O}$. Therefore, the real parts of $\tilde{S}_{f}^{2}$, the dual curve ( $\left.\tilde{e}\right)$ lying on $\tilde{S}_{f}^{2}$ and the coordinate system of $\tilde{S}_{f}^{2}$ are given by $S_{f}^{2},(e)$ and $(x, y, z)$ with origin $O$, respectively. For the real indicatrix of E. Study mapping between one-sheeted hyperboloid and $(e)$ on $S_{f}^{2}$, see Figure 1. 


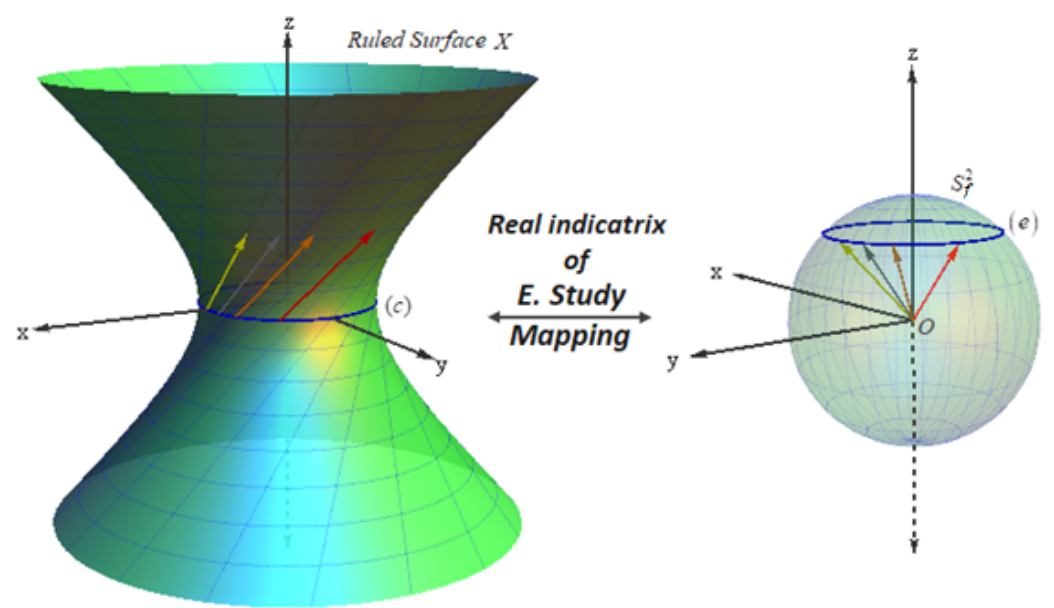

Figure 1. The real indicatrix of E. Study mapping between one-sheeted hyperboloid and $(e)$ on $S_{f}^{2}$.

Let take the parametrization of cone as

$$
Y\left(t_{m}, v_{m}\right)=(0,-1,0)+v_{m}\left(\cos t_{m}, \sin t_{m}, 1\right) .
$$

Suppose the striction curve is denoted by $\left(c_{m}\right)$. It is easy to see that $c_{m}\left(t_{m}\right)=(0,-1,0)$. Also, let denote the Frenet frame of $Y\left(t_{m}, v_{m}\right)$ as $\left\{\vec{e}_{m}, \vec{t}_{m}, \vec{g}_{m}\right\}$ along the striction curve $\left(c_{m}\right)$. Then we have

$$
\begin{aligned}
& \vec{e}_{m}\left(t_{m}\right)=\frac{1}{\sqrt{2}}\left(\cos t_{m}, \sin t_{m}, 1\right), \\
& \vec{t}_{m}\left(t_{m}\right)=\left(-\sin t_{m}, \cos t_{m}, 0\right), \\
& \vec{g}_{m}\left(t_{m}\right)=\frac{1}{\sqrt{2}}\left(-\cos t_{m},-\sin t_{m}, 1\right),
\end{aligned}
$$

where $\left\|\vec{e}_{m}^{\prime}\left(t_{m}\right)\right\|=\frac{1}{\sqrt{2}}$. When the arc-length of $\left(c_{m}\right)$ is given as $s_{m}$, we have $s_{m}=$ $\int_{0}^{t_{m}}\left\|\vec{e}_{m}^{\prime}(u)\right\| d u=\frac{t_{m}}{\sqrt{2}}$. Now, we can write the striction curve $\left(c_{m}\right)$ and $\left\{\vec{e}_{m}, \vec{t}_{m}, \vec{g}_{m}\right\}$ elements with respect to the arc-length $s_{m}$ as

$$
c_{m}\left(s_{m}\right)=(0,-1,0)
$$

and

$$
\begin{aligned}
& \vec{e}_{m}\left(s_{m}\right)=\frac{1}{\sqrt{2}}\left(\cos \sqrt{2} s_{m}, \sin \sqrt{2} s_{m}, 1\right) \\
& \vec{t}_{m}\left(s_{m}\right)=\left(-\sin \sqrt{2} s_{m}, \cos \sqrt{2} s_{m}, 0\right) \\
& \vec{g}_{m}\left(s_{m}\right)=\frac{1}{\sqrt{2}}\left(-\cos \sqrt{2} s_{m},-\sin \sqrt{2} s_{m}, 1\right),
\end{aligned}
$$

respectively. Furthermore, one can easly see that the position vector of the striction curve is $\vec{c}_{m}\left(s_{m}\right)=(0,-1,0)$.

Now, let denote the invariants of cone as $\gamma_{m}, \delta_{m}$ and $\Delta_{m}$. These invariants can be obtained as follows, respectively:

By using the derivative formulas of the Darboux frame $\left\{\vec{e}_{m}, \vec{t}_{m}, \vec{g}_{m}\right\}$, the conical curvature is obtained as $\gamma_{m}\left(s_{m}\right)=\left\langle\vec{t}_{m}^{\prime}\left(s_{m}\right), \vec{g}_{m}\left(s_{m}\right)\right\rangle=1$. Via the derivative of $\vec{c}_{m}\left(s_{m}\right)$, the other two functions are obtained as $\delta_{m}\left(s_{m}\right)=\left\langle\vec{c}_{m}^{\prime}\left(s_{m}\right), \vec{e}_{m}\left(s_{m}\right)\right\rangle=0$ and $\Delta_{m}\left(s_{m}\right)=$ $\operatorname{det}\left(\vec{c}_{m}^{\prime}\left(s_{m}\right), \vec{e}_{m}\left(s_{m}\right), \vec{t}_{m}\left(s_{m}\right)\right)=0$. By using Eqs. (4.17), the moment vectors are obtained 
as follows:

$$
\begin{aligned}
& \vec{e}_{m}^{*}\left(s_{m}\right)=\vec{c}_{m}\left(s_{m}\right) \times \vec{e}_{m}\left(s_{m}\right)=\frac{1}{\sqrt{2}}\left(-1,0, \cos \sqrt{2} s_{m}\right), \\
& \vec{t}_{m}^{*}\left(s_{m}\right)=\vec{c}_{m}\left(s_{m}\right) \times \vec{t}_{m}\left(s_{m}\right)=\left(0,0,-\sin \sqrt{2} s_{m}\right), \\
& \vec{g}_{m}^{*}\left(s_{m}\right)=\vec{c}_{m}\left(s_{m}\right) \times \vec{g}_{m}\left(s_{m}\right)=\frac{1}{\sqrt{2}}\left(-1,0,-\cos \sqrt{2} s_{m}\right) .
\end{aligned}
$$

From the Eqs. (4.17) and (4.18) the dual unit vectors correspond to cone are obtained as

$$
\begin{aligned}
& \tilde{e}_{m}\left(s_{m}\right)=\frac{1}{\sqrt{2}}\left(\cos \sqrt{2} s_{m}, \sin \sqrt{2} s_{m}, 1\right)+\varepsilon \frac{1}{\sqrt{2}}\left(-1,0, \cos \sqrt{2} s_{m}\right), \\
& \tilde{t}_{m}\left(s_{m}\right)=\left(-\sin \sqrt{2} s_{m}, \cos \sqrt{2} s_{m}, 0\right)+\varepsilon\left(0,0,-\sin \sqrt{2} s_{m}\right), \\
& \tilde{g}_{m}\left(s_{m}\right)=\frac{1}{\sqrt{2}}\left(-\cos \sqrt{2} s_{m},-\sin \sqrt{2} s_{m}, 1\right)+\varepsilon \frac{1}{\sqrt{2}}\left(-1,0,-\cos \sqrt{2} s_{m}\right),
\end{aligned}
$$

where $\left(\tilde{e}_{m}\right)$ is the dual curve on $\tilde{S}_{m}^{2}$. From (3.3), the dual curvature of cone is obtained as

$$
\bar{\gamma}_{m}=\gamma_{m}+\varepsilon\left(\delta_{m}-\gamma_{m} \Delta_{m}\right)=1
$$

We assume that $\tilde{S}_{m}^{2}$ is established in the moving coordinate system $\left(\tilde{x}_{m}, \tilde{y}_{m}, \tilde{z}_{m}\right)$ with origin $\tilde{O}_{m}$. Therefore, the real parts of $\tilde{S}_{m}^{2}$, the dual curve $\left(\tilde{e}_{m}\right)$ lying on $\tilde{S}_{m}^{2}$ and the coordinate system of $\tilde{S}_{m}^{2}$ are given by $S_{m}^{2},\left(e_{m}\right)$ and $\left(x_{m}, y_{m}, z_{m}\right)$ with origin $O_{m}$, respectively. For the real indicatrix of E. Study mapping between cone and $\left(e_{m}\right)$ on $S_{m}^{2}$, see Figure 2.

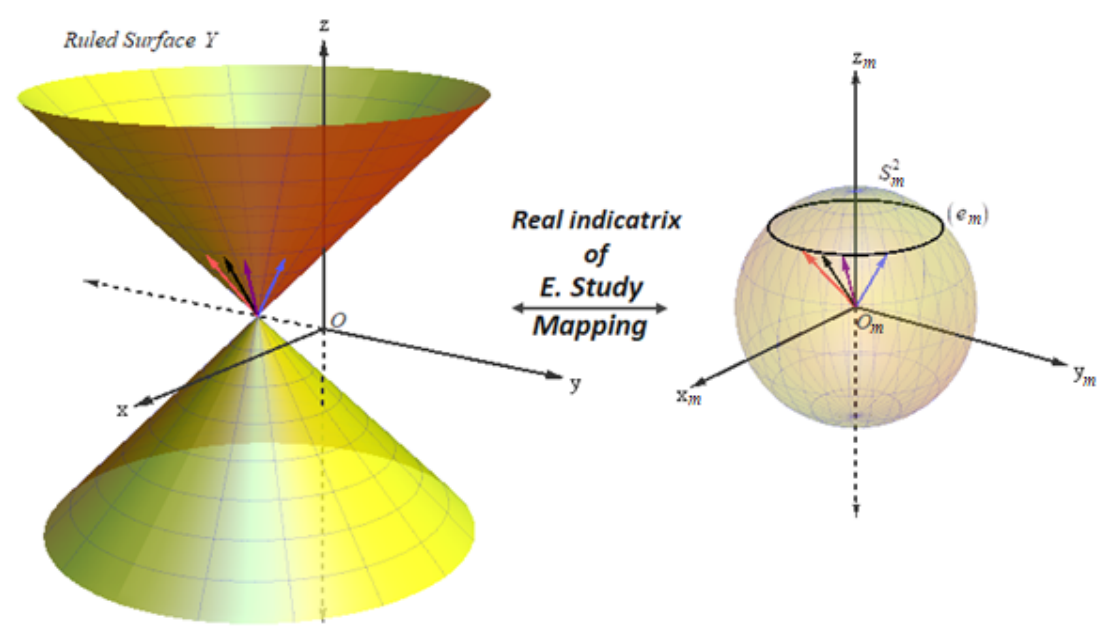

Figure 2. The real indicatrix of E. Study mapping between cone and $\left(e_{m}\right)$ on $S_{m}^{2}$.

From (4.12), the dual angular velocity of moving sphere $\tilde{S}_{m}^{2}$ rolling on fixed sphere $\tilde{S}_{f}^{2}$ is obtained as

$$
\tilde{d}=-\bar{\sigma} \bar{\gamma}_{i} \tilde{e}=2 \varepsilon \bar{\sigma} \tilde{e}
$$

where $\bar{\sigma}=\frac{d \bar{s}}{d \bar{t}}$ is the magnitude of dual rolling velocity and $\bar{\gamma}_{i}=\bar{\gamma}_{m}-\bar{\gamma}=-2 \varepsilon$ is the induced dual spherical curvature function obtained from (4.16) and (4.20).

Let $P$ denotes the contact point of coincident Frenet frames $\{\vec{e}, \vec{t}, \vec{g}\}$ and $\left\{\vec{e}_{m}, \vec{t}_{m}, \vec{g}_{m}\right\}$ along the striction curves $(c)$ and $\left(c_{m}\right)$ of one-sheeted hyperboloid and cone, respectively. Then one can see the common Frenet frame in Figure 3. 


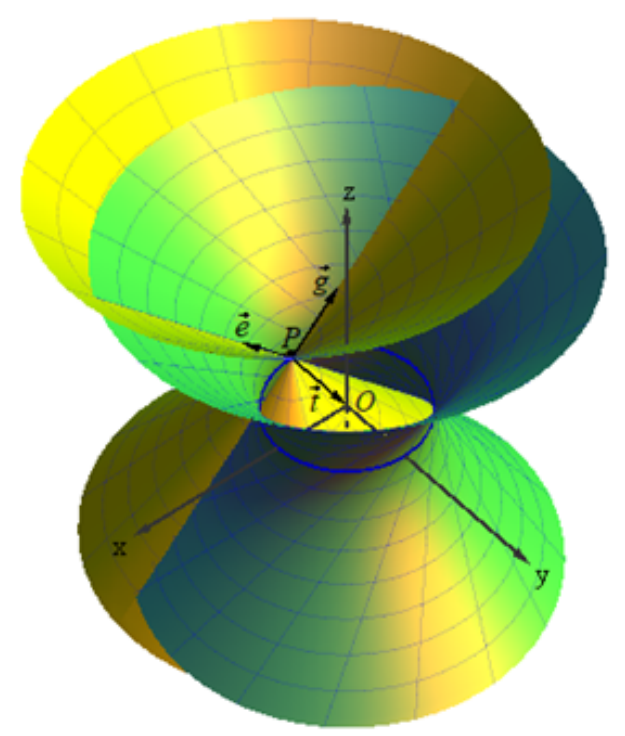

Figure 3. The common Frenet frame of one-sheeted hyperboloid and cone at $P$.

By the E. Study mapping, one-sheeted hyperboloid denoted by $X$ and cone denoted by $Y$ correspond the dual curves $(\tilde{e})$ on $\tilde{S}_{f}^{2}$ and $\left(\tilde{e}_{m}\right)$ on $\tilde{S}_{m}^{2}$, respectively. When we take the arc-length of $X$ as $\sqrt{2} s=\frac{3 \pi}{2}$ and arc-length of $Y$ as $\sqrt{2} s_{m}=0$, the dual values $\tilde{e}(3 \pi / 2)$ in (4.15) and $\tilde{e}_{m}(0)$ in (4.19) become identical and then we obtain the dual contact point $\tilde{P}=P+\varepsilon P^{*}$ as $\tilde{P}=\frac{1}{\sqrt{2}}(1,0,1)+\varepsilon \frac{1}{\sqrt{2}}(1,0,-1)$. Figure 4 shows the real parts of $\tilde{S}_{f}^{2}$ and $\tilde{S}_{m}^{2}$, dual curves, dual Darboux frame and dual coordinate systems.

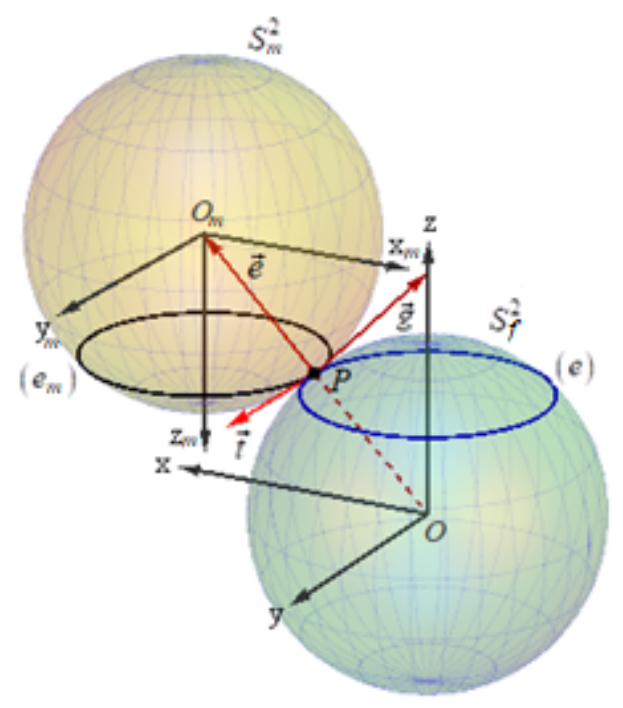

Figure 4. The real indicatrix of dual rolling contact motion.

Consequently, Figure 3 and Figure 4 show the relations between the common Frenet frames and real indicatrix of dual Darboux frames at the contact point $P$. 


\section{Conclusion}

In this paper, we study the forward kinematics of dual rolling contact motion between the moving and fixed dual unit spheres $\tilde{S}_{f}^{2}$ and $\tilde{S}_{m}^{2}$ through their dual curves $(\tilde{e})$ and $\left(\tilde{e}_{m}\right)$ by adopting the dual Darboux frame in dual space. We obtain a new equation of the dual angular velocity of moving sphere $\tilde{S}_{m}^{2}$. The new formulation is specified with respect to three dual unit vectors and dual geometric invariants, which are dual arc lengths of the dual contact curves and the dual induced curvature of the two spheres.

\section{References}

[1] P. Azariadis and N. Aspragathos, Computer graphics representation and transformation of geometric entities using dual unit vectors and line transformations, Comput. Graph. 25 (2), 195-209, 2001.

[2] W. Blaschke, Differential Geometrie and Geometrischke Grundlagen ven Einsteins Relativitasttheorie, Dover, New York, 1945.

[3] O. Bottema and B. Roth, Theoretical Kinematics, North-Holland Publ. Co., Amsterdam, 1979.

[4] H.H. Cheng, Programming with dual numbers and its applications in mechanisms design, Eng. Comput. 10 (4), 212-229, 1994.

[5] W.K. Clifford, Preliminary sketch of biquaternions, Proc. Lond. Math. Soc. 4 (64), 381-395, 1873.

[6] L. Cui and J.S. Dai, A Darboux-frame-based formulation of spin-rolling motion of rigid objects with point contact, IEEE Trans. Rob. 26 (2), 383-388, 2010.

[7] F.M Dimentberg, The Screw Calculus and its Applications in Mechanics, English translation: AD680993, Clearinghouse for Federal and Scientific Technical Information, (Izdat. Nauka, Moscow, USSR), 1965.

[8] A. Karger and J. Novak, Space Kinematics and Lie Groups, Gordon and Breach Science Publishers, Prague, 1978.

[9] A.P. Kotelnikov, Screw Calculus and Some Applications to Geometry and Mechanics, Annals of Imperial University of Kazan, 1895.

[10] J.M. McCarthy, Geometric Design of Linkages, Springer, 2000.

[11] J.M. McCarthy and B. Ravani, Differential kinematic of spherical and spatial motions using kinematic mapping, J. Appl. Mech. 53 (1), 15-22, 1986.

[12] H.R. Müller, Kinematik Dersleri, Ankara Üniversitesi Fen Fakültesi Yaynlar, 1963.

[13] E.W. Nelson, C.L. Best and W.G. McLean, Schaums Outline of Theory and Problems of Engineering Mechanics, Statics and Dynamics, (5th Ed.), McGraw-Hill, New York, 1997.

[14] E. Study, Geometrie der Dynamen, Leibzig, 1903.

[15] H.H. Uğurlu, The principle of transference between real and dual Lorentzian spaces and dual Lorentzian angles, 16th International Geometry Symposium, Celal Bayar University, Manisa, Turkey, 2018.

[16] G.R. Veldkamp, On the use of dual numbers, vectors and matrices in instantaneous spatial kinematics, Mech. Mach. Theory, 11 (2), 141-156, 1976.

[17] A.T. Yang and F. Freudenstein, Application of dual number quaternions algebra to the analysis of spatial mechanisms, J. Appl. Mech. 31 (2), 300-308, 1964.

[18] X.F. Zha, A new approach to generation of ruled surfaces and its applications in engineering, Int. J. Adv. Manuf. Technol. 13 (3) 155-163, 1997. 